\title{
Are substitutes to Cd-based quantum dots in displays more sustainable, effective, and cost competitive? An alternatives assessment approach
}

\author{
Aude Bechu ${ }^{\dagger}$, Subhasis Ghoshal ${ }^{\S}$, Audrey H. Moores ${ }^{\dagger \#^{*}}$, Niladri Basu ${ }^{\ddagger *}$ \\ ${ }^{\dagger}$ Department of Chemistry, McGill University, Montreal, Quebec H3A OB8, Canada \\ $\S$ Department of Civil Engineering, McGill University, Montreal, Quebec H3A 0C3, Canada
}

\pm Centre for Green Chemistry and Catalysis, Department of Chemistry, McGill University, Montreal, Quebec H3A 0B8,

Canada

\#Department of Materials Engineering, McGill University, Montreal, Quebec H3A 0C5, Canada

‡Faculty of Agricultural and Environmental Sciences, McGill University, Ste. Anne de Bellevue, Quebec H9X 3V9, Canada

\begin{abstract}
:
Light emissive organics and inorganic nanoparticles are substance classes competing for applications in displays in the form of organic LEDs (OLEDs) and quantum LEDs (QLEDs), respectively. Upcoming substance classes (perovskites) and Q-OLED displays also contain novel nanomaterials and organics for these applications. However, the sustainability of these emissive substances is difficult to assess quickly and broadly because of their complexity, their inherently different structures, and their rapid evolution in the literature. We propose the use of an alternatives assessment to compare the hazard, cost, and performance of these competing substances, with a focus on replacing cadmiumcontaining quantum dots. The cost assessment highlights competitiveness of OLEDs because of their low amounts needed per display, but performance assessments do not identify a preferred alternative. The hazard results indicate there is no clear alternative either, with each novel nanomaterial or organic substance having different negative aspects. These results identify the need for a low-hazard highperforming alternative substance, and the assessment provides a framework for researchers to evaluate their own novel substances.
\end{abstract}




\section{Introduction:}

Globally, the fate of $83 \%$ of electronic waste (E-waste) is unknown, likely dumped or dismantled in potentially hazardous circumstances. ${ }^{1}$ This undocumented E-waste (44 million metric tons generated in 2019 alone) has embedded chemicals and substances that are commonly released into the environment. ${ }^{1}$ One type of electronic good with increasingly diverse chemicals are screens and monitors, which currently make up 12.5 wt\% of global E-waste. Contrary to global E-waste trends, the total weight of disposed screens and monitors is decreasing slightly (by $1 \%$ over 4 years). This shift is attributed to the replacement of traditional heavy cathode ray tube (CRT) monitors with lighter flat panel displays in waste streams. ${ }^{1}$ These two screens rely on different technologies and heavy metals, with CRT relying heavily on lead while flat screens have a cocktail of heavy metals. ${ }^{2}$ This technology shift has represented an overall decrease in certain end-of-life impacts, ${ }^{2}$ although unknown risks remain.

The next generation of flat screen televisions currently has many different emissive substance classes vying for dominance. ${ }^{3}$ These classes use novel substances that convert charge or light into specific colors. The main substance of concern is cadmium-containing quantum dots (Cd-QDs), due to the known toxicity of $\mathrm{Cd} .{ }^{4}$ This substance of concern has led to numerous studies reviewing the sustainability of the Cd-QDs and competing substances. ${ }^{5}$ For example, life cycle assessments (LCAs) have been performed to compare the cumulative energy demand of Cd-QDs and indium-containing QDs (In-QDs). ${ }^{6}$ After updating these assessments with new data on the amount of materials in televisions, In-QDs were found to require approximately 13 times more energy $\left(\mathrm{MJ} \mathrm{cm}^{-2}\right)$ than $\mathrm{Cd}-\mathrm{QDs} .{ }^{7}$ LCAs have also been used to point out areas of concern in the manufacturing of Cd-QDs into quantum light emitting diode displays (QLEDs), such as the aquatic acidification and ecotoxicity of a Cd-QD encapsulating polymer. ${ }^{8}$ Another substance class of interest to displays, perovskites, have attracted significant research attention for their use in photovoltaic panels, and sustainability assessments have mostly focused on that application. Studies have weighed the risks of using lead in perovskites versus lead alternatives using a variety of metrics, with some concluding that lead-based perovskites performance outweighs the possible risk, ${ }^{9}$ while others encourage the development of lead-free materials. ${ }^{10}$ Lastly, QLEDs are functionally matched by organic LEDs (OLEDs), which rely on specific organic emitters (OEs). In sustainability assessments, however, OLEDs are commonly compared to traditional liquid crystal displays (LCDs). OLEDs require less energy over their lifetime, ${ }^{11}$ but assessments focused on the end of life of these materials caution the higher toxicity 
potential. ${ }^{12}$ In summary, these Cd-QD, In-QD, perovskite and OLED assessments have pointed out sustainability concerns unique to each substance class.

The sustainability studies mentioned above employ LCA, which has provided in-depth and useful comparisons between two substance classes. However, there are challenges with LCA in assessing all emissive substances that are still at the research stage. ${ }^{13}$ For example, modelling a scale-up scenario is time consuming and involves various assumptions to cover missing impact data. ${ }^{13}$ In addition, the toxicity profiles of these research-stage nanomaterials of complex composition are not often known and cannot be easily predicted by models. ${ }^{13}$ These challenges, and many others, hinder the use of LCAs for nanomaterials at the research stage. ${ }^{14,15} \mathrm{~A}$ high-level yet simple sustainability evaluation is needed that encompasses all substance classes while remaining accessible to researchers developing these substances.

The alternatives assessment framework was developed to help identify safer and effective alternatives to a chemical of concern. ${ }^{16,17}$ This methodology prioritizes reducing the potential harm of new substances, while also recognizing only the alternatives that are feasible (e.g. in terms of cost and performance). It is a flexible assessment that allows for comparison of numerous options and materials classes if desired. For example, Gilbertson and Ng compared organics to minerals to nanomaterials in a search for alternatives to brominated fire retardants. ${ }^{18}$ We have previously published an alternatives assessment on 46 perovskites in solar cells, which has identified the least hazardous alternative as well as the steps needed for that alternative to be more competitive with the highest-performing lead-based material. ${ }^{19}$ Alternatives assessment are powerful methods in the context of sustainable chemistry because they enable research on the design and synthesis of safer alternative molecules and materials, but also a simple assessment of emerging one. ${ }^{20}$ We hypothesize that this flexible assessment could also be used by substance researchers to identify responsible replacements for $\mathrm{Cd}-\mathrm{QDs}$ for displays. This assessment could then identify promising substance classes that need further investigation via LCA.

The objective of this study is to analyze Cd-QDs and its possible replacement substance classes with an alternatives assessment to foster a broad understanding of the field. We followed the US National Research Council guidelines ${ }^{17}$ and made the assessment fit for the purpose of analyzing emissive substances in displays. We produced three evaluations: performance, cost, and hazard. Using substance syntheses and reported performance metrics as inputs, we output a score in each evaluation. Then, we compared the scores of different substance classes with the goal of finding a viable substitution. We also aimed to structure this alternatives assessment so that materials researchers can add in their own 
chemicals (in a reasonable timeframe) and compare them to other emerging substance classes. In short, an alternatives assessment for emissive substances can help point researchers to an informed substitution of Cd-QDs.

\section{Methods}

This assessment follows the steps of the US National Academy's Framework to Guide Selection of Chemical Alternatives. ${ }^{17}$ Steps 1-4 are outlined in the following section "Identify substance class of concern \& scoping problem formulation". Step 5-7 and 9 encompass the Cost, Performance and Hazard assessments sections of the methods. Step 8 involved integrating life cycle analysis, which we did not pursue due to the early research stage (and therefore high uncertainty) of some analyzed substances. Step 10 and 11 involves bringing together all evaluations to compare and identify an acceptable alternative. These steps are addressed in the "Multiparameter Evaluation" section. All steps and methods sections are represented schematically below (Figure 1), and details will be reported in the following sections. 
General NAS framework steps (left) applied to emerging emissive substances (right)

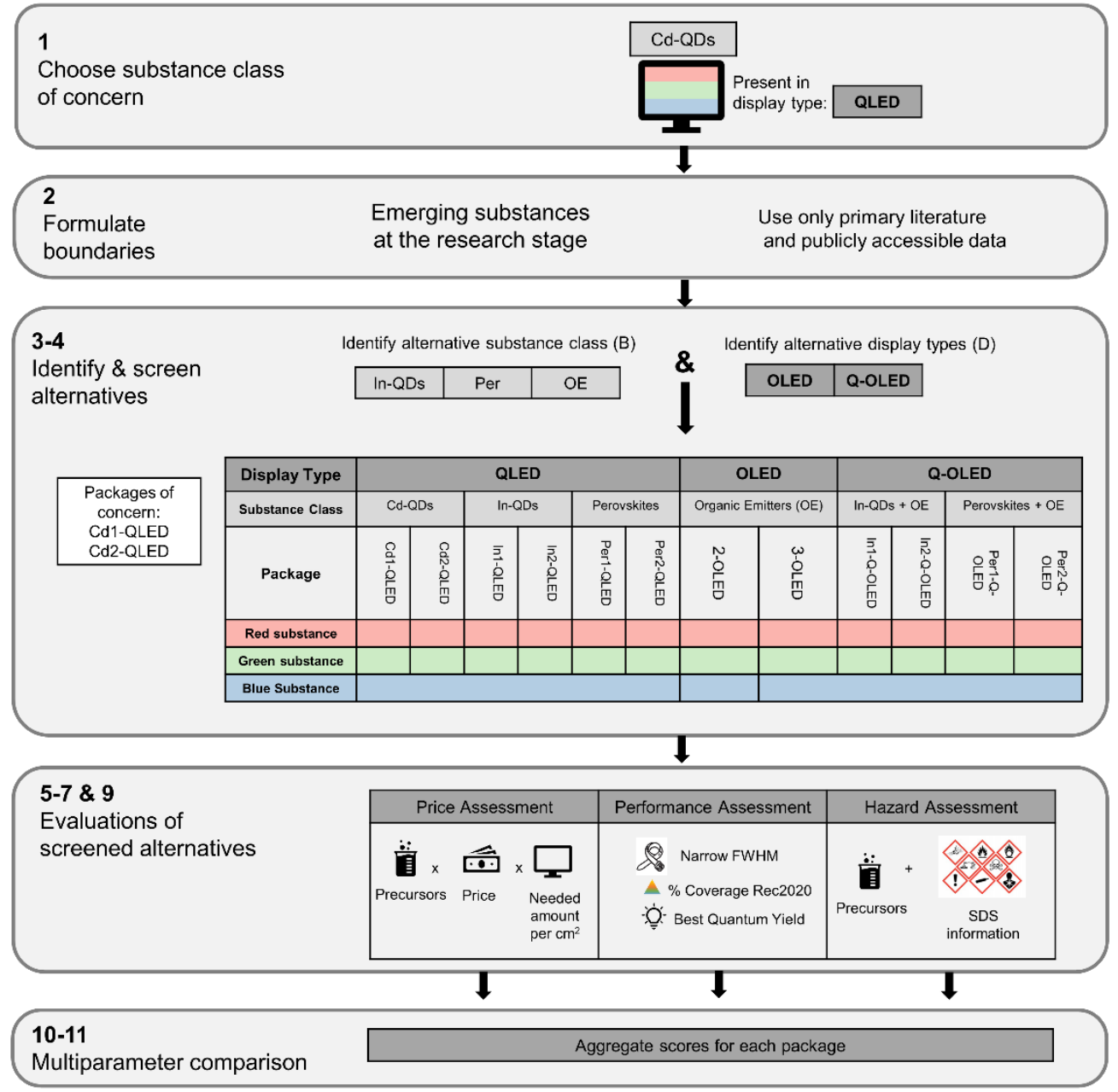

Figure 1: Outline of substance class of concern and alternatives packages examined in this alternatives assessment. Step 1 identifies the substance class of concern, Cd-QDs, which are integrated into displays. Step 2 points out two different boundaries employed in this assessment. Four key terms are bolded in the large table of steps 3-4: substances, substance class, packages, and display types. These terms are all significant to categorizing alternatives. Steps 5-7 \& 9 illustrate the three different evaluations conducted for each package: price, performance, and hazard. Steps 10-11 indicate that these three evaluations will be aggregated into one score for each package.

Identify substance class of concern \& scoping problem formulation 
The first four steps of this framework involve (1) choosing a substance of concern, (2) formulating the boundaries of the assessment (3), identifying alternatives, and (4) screening the alternatives. We decided to abide by the following three decision rules as boundaries to the assessment. First, the foundational data for this assessment is based on public knowledge. Second, the alternatives presented are emerging substance classes, which are highlighted to spur development. Third, the scaling up of the production of these alternatives is not considered.

The four steps mentioned above were informed by a search of both the scientific literature and press releases from display and QD companies. Literature was searched before April 2021 and considered for use in this assessment if it met the following criteria: (1) use of developed substance in a device; and (2) explicitly outlined steps and procedures in chemical and device fabrication. Patents were excluded from the search. We also consulted with scientists developing these substances as well as those assessing the degradation and subsequent toxicity of these substances through the McGill Sustainability Systems Initiative (MSSI).

As noted in the introduction, we aim to identify responsible replacements of Cd-QDs in displays using alternatives assessment. We defined Cd-QDs as a substance class that is made up of many substances (different Cd-containing QDs from different syntheses). We chose two different syntheses of cadmium-containing quantum dots, therefore having $\mathrm{Cd} 1-\mathrm{QDs} \mathrm{s}^{21}$ and $\mathrm{Cd} 2-\mathrm{QD}^{22}$ substances. We chose two syntheses to demonstrate possible differences inherent to one substance class (in the case of QDs there are different shell compositions and precursors). We chose these two syntheses specifically because they both produce high performing Cd-QDs.

To connect these substances to their end use in displays and rely on uniform nomenclature, we organized the assessment according to four key designations (highlighted as A-D in this paragraph, also displayed in Scheme 1). (A) Substances are individual single or multiple mineral phases or organic compounds that achieve a desired function (emitting red, green, or blue light in this case). Substances belong to a (B) substance class. A substance class is composed of substances that are generally similar in structure. For example, Cd-QDs are a substance class. Returning to (A) substances, we chose to group red, green, and blue emitting substances together because they make up the three colored pixels in displays (Table S1). Each such group was designated as a (C) package. These packages will essentially be the units analyzed in this alternatives assessment. Lastly, there are (D) display types. We decided to integrate display types because these are known by consumers and marketed by the industry. The Cd-QDs 
substance class is used in a display type (QLEDs). QLEDs have blue substances that emit light from charge, and red and green substances that emit light by down-converting blue light.

According to the above definitions, Cd-QD substance class cannot make up a package on its own because it must have a blue emissive material. To complete the package, we added InGaN substance as a blue emitting material. ${ }^{23,24}$ InGaN also provides the blue backlight necessary for QDs, which down-convert the light. ${ }^{25}$ In GaN is therefore an integral part of the display type, QLED. In summary, the substance class of concern was grouped into two packages: Cd1-QLED and Cd2-QLED. These packages are labelled such a way that the display type (QLED) and the substance class (Cd-QDs) can be identified. The 1 and 2 refer to different synthetic methods for slightly different chemical compositions of the substances (Cd1-QDs and Cd2-QDs).

\section{Identify and screen alternatives}

In a similar manner as the substance class of concern, alternative substance classes were grouped in packages of red, green, and blue emissive materials. Alternatives to Cd-QDs were chosen to represent three different emerging substance classes: In-based QDs, perovskites, and organic emitters (OEs). Chosen alternative substances were high performers in their respective substance classes (see SI excel sheet for complete list of alternatives screened). Performance was based on high quantum yield (QY) and external quantum efficiency (EQE), both of which indicate that the material is adept at transforming light or charge into a given wavelength.

In-based QDs are currently replacing Cd-based QDs in some displays because of their lack of Cd, due to the latter's toxicity concerns. The synthesis of In-based QDs is less mature than that of Cd-based QDs, and was challenged by initially with a lower quantum yield and broader emission. ${ }^{25}$ Two In-based QD substances were explored, and since they need an InGaN backlight (and therefore QLED display type), they are identified as In1-QLED ${ }^{26}$ and In2-QLED. ${ }^{27}$

Perovskites are presented in literature as having a similar emissive tunability as QDs, but with simpler syntheses. These materials are promising because of their defect tolerance and high rates of emission, but the development of a blue material lags behind green and red ones. ${ }^{28}$ Two green/red perovskite alternative substances were chosen, one which contains lead, ${ }^{29}$ while the other is lead-free. ${ }^{30}$ Although the lead-based perovskite alternative has high performance, we anticipate the presence of lead could create a similar situation as $\mathrm{Cd}$, as both elements are heavy metals with known adverse health 
effects covered by the same EU regulations. ${ }^{31}$ These perovskites also retain the InGaN backlight, and are identified as Per1-QLED ${ }^{29}$ and Per2-QLED. ${ }^{30}$

OEs are currently being developed as alternative substances and have the advantage of generating all colors from charge, rather than needing a blue color excitation which must be down converted. This means that OEs have their own display type: OLEDs. OEs are a rapidly evolving field, but recent developments can be approximately categorized according to their method of exciton recombination: phosphorescence (Ph) and thermally activated delayed fluorescence (TaDF). ${ }^{32}$ These categories are thoroughly explored in several reviews ${ }^{33-35}$, but we chose to focus on the best-performing materials identified by Bräse et al. ${ }^{32}$ For OEs, a green, red, and blue chemical substance of each generation were chosen for packages 2-OLED ${ }^{36-38}(\mathrm{Ph})$ and 3-OLED ${ }^{39-41}(\mathrm{TaDF})$. It is unclear which alternative substance is used by industry, but we decided to investigate these because of their impressive performance in academic papers.

Lastly, we looked ahead to upcoming market-ready developments (which we could emulate here) to showcase the versatility of the alternatives assessment approach. The development of Q-OLEDs aimed to address separate issues with QLED and OLED display types by combining different substance classes. ${ }^{42}$ This substance class promises "lower cost, higher brightness, improved power efficiency and more accurate color reproduction". ${ }^{42}$ In this next generation substance class, blue OEs substances are matched with emerging green and red substances. ${ }^{42}$ For our assessment, we consider these emerging substances from the In-QDs or perovskites substance classes. This creates the following alternative packages: In1-QOLED, ${ }^{26,41}$ In2-Q-OLED, ${ }^{27,41}$ Per1-Q-OLED, ${ }^{29,41}$ and Per2-Q-OLED. ${ }^{30,41}$

In short, there are 12 packages evaluated in this assessment (see Table S1 for summary). There are 2 packages of known concern, Cd1-QLED and Cd2-QLED. There are 4 packages of alternative QLED displays based on In-QDs (In1-QLED and In2-QLED) and perovskites (Per1-QLED and Per2-QLED) substance classes. There are 2 packages based on the alternative OLED display types which use OEs (2OLED and 3-OLED). Then there are 4 packages of Q-OLED display types, which use a combination of OEs for blues and either In-QDs or perovskites for green and red (In1-Q-OLED, In2-Q-OLED and Per1-Q-OLED, Per2-Q-OLED). In total, these 12 packages span different display types and substance classes.

\section{Price Assessment}

We followed the method set up by Chen et $a .^{27}$ to estimate the costs of each alternative package. The amounts of each alternative substance was calculated based on the needs to cover $1 \mathrm{~cm}^{2}$ of screen area, and then added to the cost of the other substances in the package. This calculation involved 
finding the cost of each chemical used in the synthesis, estimating the yield of the synthesis, and determining how much of each chemical is used in $1 \mathrm{~cm}^{2}$ of screen area. These three steps each involved certain assumptions described below.

After the precursors of each substance were identified, their cost was found by searching for the chemical on Sigma Aldrich 2021 catalogue and choosing the price (\$CAD) of the largest container size (up to $1 \mathrm{~kg}$ for solids or $4 \mathrm{~L}$ for liquids). The purity examined matched that described in the syntheses. The costs of manufacturing different alternatives were not considered due to the large differences between small-scale syntheses and industrial operations.

After the price of each precursor was established, the final price of the alternative material was estimated based on certain yields. The yields of OLED materials are stated in the literature, ${ }^{36-41}$ however the yield of QDs and perovskites are not stated in the cited papers. ${ }^{21,26,29,30,43,44}$ To account for this, we averaged the yield in all steps of the OLEDs synthesis and applied this yield to the limiting reagent in the QDs and perovskites syntheses (see SI spreadsheet for each calculation). For InGaN, the reagents were chosen based on fundamental knowledge of the syntheses ${ }^{24,45}$ and the same OLED-based yield was applied (no one limiting reagent).

With the cost of $1 \mathrm{~kg}$ of each alternative material calculated, costs were combined with estimated costs of other materials into a given package (of red, green, and blue materials, see Table 1). We assumed QDs concentrations from concentrations of $\mathrm{Cd}$ and In from commercial televisions. ${ }^{7}$ We assumed that perovskites would be present in displays at the same concentrations as Cd-QDs. For OLEDs, we assumed that the amount of In present aligned with estimates by Zink et al. ${ }^{46}$ We also assumed that In-free OLED alternatives would be present in the same molar concentration as In-containing OLEDs. For InGaN, we chose the amount necessary in a display based of a detailed life cycle assessment. ${ }^{24}$

\section{Performance Assessment}

Successful materials for color generation in television must meet a variety of performance requirements. Two favorable characteristics are widely reported; ${ }^{22,44,47}$ photoluminescence quantum yield (QY) and narrow emission spectra as quantified by full width at half maximum (FWHM). We chose these as two out of three performance metrics.

In addition, it is key to determine if the material produces the exact wavelength necessary for displays. ITU-R Recommendation 2020 (Rec. 2020) color standard is the benchmark of colors possible in a 
display based on a combination of the ideal red, green and blue pixels. ${ }^{48}$ To calculate whether a material meets this standard, the peak wavelength of emitted light was converted to $X, Y$ space in the CIE color graph (Commision Internationale de L'Eclairage). These $(X, Y)$ values were then plotted on a graph and the overlap with Rec. 2020 color standard was calculated (see online tool ${ }^{49}$ and SI for details). Although there are numerous examples of $>90 \%$ coverage of Rec. 2020 with certain combinations of QDs, ${ }^{50}$ and perovskites, ${ }^{48} 100 \%$ coverage has not yet been achieved (to the best of the our knowledge).

These three metrics (quantum yield, FWHM, and percent coverage Rec 2020) were collected for each material from their respective sources. InGaN performance information was gathered from the Ullman Encyclopedia of Industrial Chemistry ( $Q Y$ and peak wavelength) ${ }^{23}$ as well as the review by T. Wang (FWHM) ${ }^{51}$

For each package, the red, green, and blue values for each metric were averaged. For example, an alternatives' FWHM values for red $(30 \mathrm{~nm})$, green $(50 \mathrm{~nm})$ and blue $(60 \mathrm{~nm})$ were combined such that the FWHM value for the alternative package would be $46.7 \mathrm{~nm}$. Then, each averaged metric for each package was compared, with the best value ranked as 1 and the worst 0.1. For quantum yield and percent coverage Rec 2020, the highest percentages were considered best, while for FWHM, the lowest value was considered best. Then, each metric score was added together to obtain the final performance score. For example, if a package had the most Rec 2020 coverage (rank =1), the highest quantum yield $($ rank $=1)$ and the lowest FWHM (rank = 1), then its combined performance score would be 3.

\section{Hazard Assessment}

Hazard assessments are difficult to construct due to lack of data for most chemicals in commerce. Following a study by Llanos et al., ${ }^{19}$ precursor substances were selected as the inputs to the hazard assessment. This aligns with certain chemical regulations, which determine the inherent hazard of a product from its chemical inputs at the manufacturing stage, rather than possible end of life issues. ${ }^{52}$ Hazard data was obtained using the Toxics Use Reduction Institute's Pollution Prevention Options Analysis System Tool. ${ }^{53}$ This tool process data from Safety Data Sheets and the GHS into subcategory and category scores for different hazards for each chemical (See SI excel sheet for full list of subcategories and categories). The most hazardous score for each subcategory was 10 , the least hazardous was 2 , and if data was missing, we entered in 0 (see SI excel sheet for full assessment). The worst variable in each subcategory score of the P2OAsys analysis was used as the final subcategory score. The score of each category was the average of the subcategory scores. The most common assessors of hazard present in the Safety Data Sheets studied were combined into three metrics; Human Health, Environment, and 
Physical Properties (see SI Table S2 for category combinations for each). In short, human health encompasses acute and chronic effects on humans. The environment metric covers the acute and chronic effects on aquatic organisms, as well as persistence and bioaccumulation potentials. Physical properties covers flammability, reactivity, corrosivity, and volatility.

\section{Multiparameter Evaluation}

The cost, performance and hazard assessments were combined to highlight the overall feasibility of the packages. This was done by first ranking the scores of the packages per evaluation on a scale of 1 (best) to 0.1 (worst). The best scores were awarded to the most cost attractive, the highest performing and the safest. A theoretical package, that scored best in all evaluations, would score a $3(1+1+1)$ in the multiparameter evaluation.

\section{Results and Discussion:}

\section{Cost assessment}

Figure 2 presents the cost in Canadian dollars of each package per $\mathrm{cm}^{2}$ of display area. These costs vary dramatically, from $\$ 1.30$ to $\$ 0.001 \mathrm{CAD} / \mathrm{cm}^{2}$ (Cd2-QLED and 3-OLED, respectively). The cost of each individual material was not correlated to the number of steps in the synthesis or the number of chemicals involved in the synthesis (Fig. S1). We acknowledge that since the cost estimates were based on the Sigma Aldrich catalog, these costs do not reflect the savings that manufacturers can achieve by buying chemicals in bulk or at slightly lower purity. However, we hypothesize that the trends in different costs of packages will persist due to the large cost differences (Figure 2). 

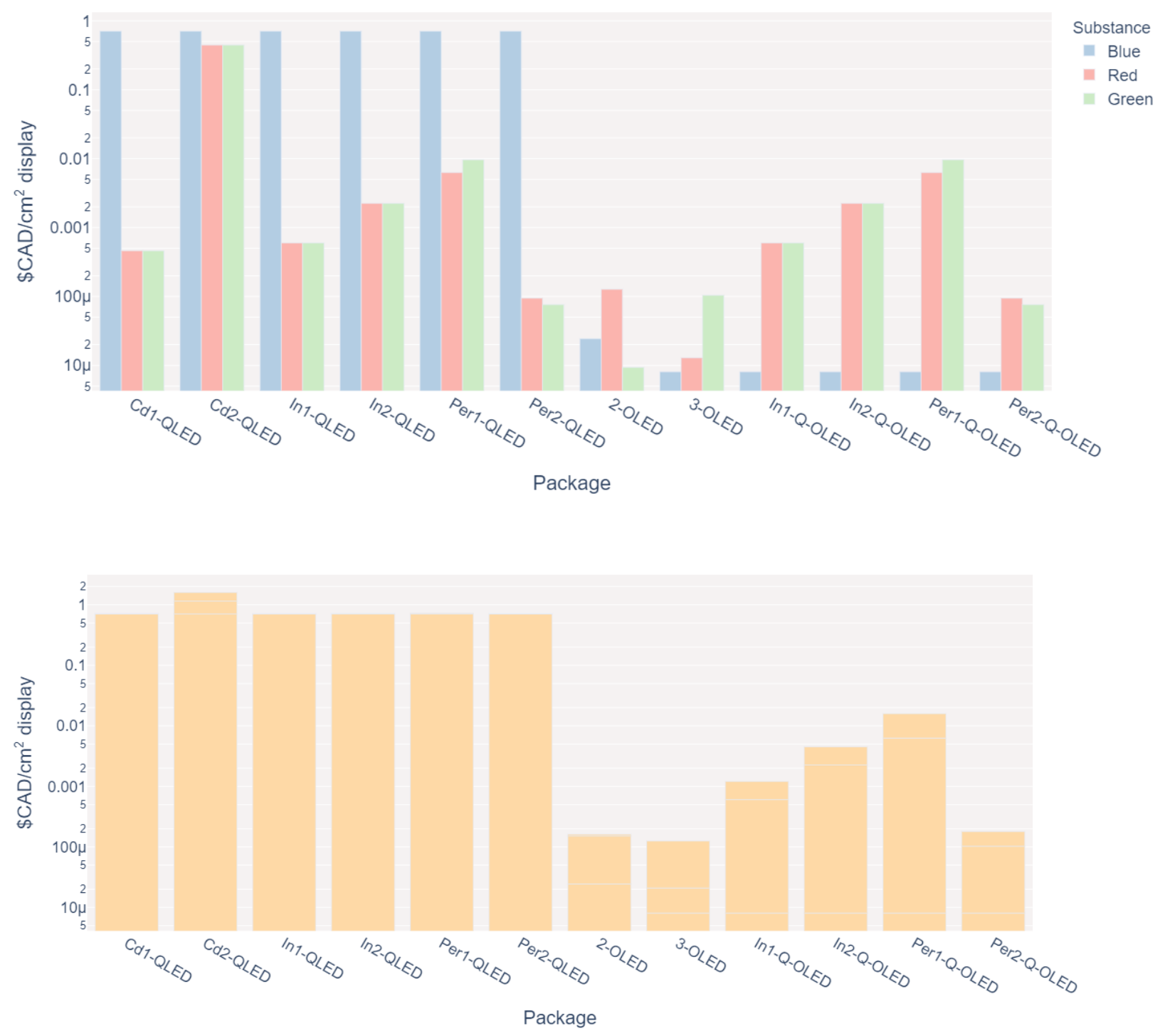

Figure 2: (A) Price assessment results, grouped by package of red, green, and blue pixels with a logarithmic $y$-axis. (B) Total prices of the packages, again on a logarithmic $y$-axis (see spreadsheet for table of values).

Most of the cost incurred by Cd2-QLED (and other QLEDs) arises from the blue emissive material, InGaN. This material is costly to produce because its precursors are costly deposition-grade metal organics (e.g. trimethylindium). In addition, the amount of InGaN per television is $~ 20 x$ more than the next highest amount of chemical (Red-Cd2-QLED) and $70,000 x$ more than the least amount of chemical 
needed (Blue-3-OLED). Therefore, improvements in both the type or precursors and the amount of BQLED would decrease the cost of the material. It is unclear whether efforts to introduce micro-LEDs ( $\mu$ LEDs) with smaller area backlights of InGaN will reduce the total amount and cost of InGaN used in the product.

OLED materials (including the B-Q-OLED) have the advantage of needing 100x less mass of material compared to pervoskites (Per) and InP QDs. These lower material requirements absorb the high cost per gram of OLEDs precursors. Such estimates of material requirements have been amended in the past due to examination of actual products. ${ }^{7}$

Figure 2 also demonstrates differences between specific procedures making the similar materials. For example, Cd1-QDs costs $>0.01 \$ C A D / \mathrm{cm}^{2}$ of display, while $C d 2-Q D$ s costs $0.60 \mathrm{CAD} / \mathrm{cm}^{2}$ of display. These differences do not lie in the amounts of materials in the television, but rather in the amount and price of the precursors compared to the yields of the syntheses. Most (80\%) of the cost of Cd2-QDs is driven using trioctlyamine, a solvent which is 10x more expensive than the solvent used in Cd1-QDs (octadecene). In addition, Cd2-QDs uses $\sim 3 x$ as much solvent as Cd1-QDs. Another example of a cost difference in the same material is between Per1-Green and Per2-Green. Specifically, Per2-Green uses much less solvent than Per1-Green $(13 \mathrm{~mL}$ octadecene and $990 \mathrm{~mL}$ octane per gram of substance, respectively), which is reflected in the price (18 and 1,514 \$CAD/g substance, respectively).

We acknowledge that a key factor in the cost assessment is missing: the cost of encapsulation. In other words, the cost of other layers in a display is not included, even though certain layers may change depending on the package employed. For example, blue OLED materials need to be more encapsulated than blue QLED, ${ }^{54}$ which increases costs.

In addition, the cost of manufacturing the materials at scale is not considered because these substances were analyzed at the lab scale. The cost of manufacturing could increase if there are many steps involved in a substance synthesis. For example, QDs need purification between core synthesis and shelling steps. ${ }^{21,22}$ The cost of manufacturing may also increase due to the inherent instability of certain substances. For example, perovskites are air and water sensitive, ${ }^{55}$ which could call for more specialized equipment at the manufacturing stage.

Lastly, a low cost does not necessarily equate to an available material on the market. For example, the EU designated certain raw materials that were economically important, but had a high supply risk in their list of Critical Raw Materials. A few elements present in this list are also key to 
substances mentioned here: Gallium, Indium, Phosphorous, Iridium. ${ }^{56}$ The substance classes that have a high supply risk are therefore In-QDs and certain OEs. In addition, In and Ga are the backbone to the blue substance in QLEDs, InGaN.

\section{Performance assessment}

The best performers had low FWHM, high quantum yield, and high Rec. 2020 overlap (e.g. Cd2QLED, Figure 3). The three highest ranking packages were Cd2-QLED, In2-QLED and Per1-QLED. These three packages had favorable (i.e. low) FWHM and high Rec 2020 overlap.

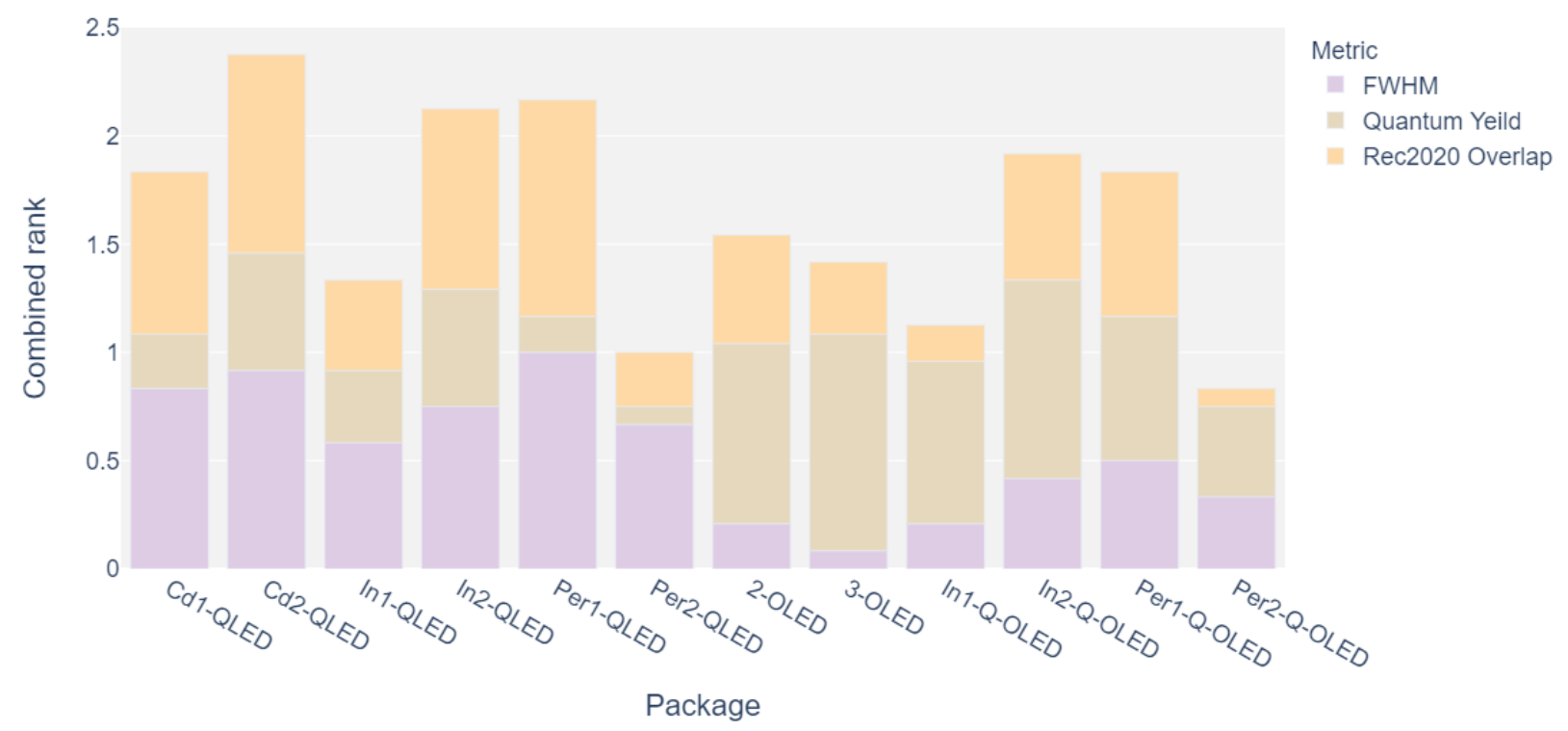

Figure 3: Performance Assessment results, grouped by FWHM (purple), Quantum Yield (brown) and Rec 2020 overlap (yellow). The highest rank possible for each performance indicator is 1 and the lowest is 0.1.

These linear rankings have the possibility of obscuring non-linear trends in each of these performance metrics. However, we observed that the trends of ranking vs. performance metric were relatively linear $\left(R^{2}=0.93-0.98\right.$, see Fig. S3). In other words, these rankings do not hide a large disparity between packages, substance classes or display types.

However, the high performance of one package was not linked to high performance of the other package of the same substance class. For example, In2-QLED performed well overall (score of 2.2), but 
In1-QLED did not perform as well (score of 1.4). Different syntheses led to very different performance scores.

For QDs or perovskites, we propose that the simplest improvement to overall performance could be made by improving the Rec. 2020 overlap. The needed wavelengths to meet this standard ${ }^{57}$ could be reached by these substance classes by changing particle size $(Q D s)^{58}$ or elemental ratios (perovskites). ${ }^{29}$ For OEs, the tuning of emission is reliant on tuning ligands. ${ }^{59}$

The performance assessment also highlights that there are differences between OLEDs and QLEDs. Blue-OLEDs have higher quantum yields and worse (i.e., higher) FWHM values than the BlueQLEDs (which are InGaN based). In addition, the Blue-3-OLED (which is also the blue color in Q-OLEDs) has an emission at $480 \mathrm{~nm}$, which lowers the Rec.2020 overlap (Fig. S2). These FWHM and emission peak (both attributed to the blue colors) decreased the overall scores of the Q-OLEDs. This need for improvement in the blue emitters is highlighted by others as well. ${ }^{28,60}$

The lowest performing packages were Per2-QLED and Per2-Q-OLED. These lead-free perovskites had low quantum yields and low overlaps with Rec. 2020. These design considerations are necessary to produce viable lead-free perovskite alternatives. Although only one lead-free perovskite alternative was explored here, other lead-free perovskite alternatives have been compared elsewhere for solar applications. ${ }^{19}$ These perovskite variations may be applicable to displays as well.

This performance assessment did not encompass all possible measures of performance. Low performance in metrics not measured here could lead to serious issues as substance classes are evaluated for implementation at scale. One such performance metric not analyzed here is stability. For each substance class, we found that researchers experienced issues with the stability of the substances. 55,61,62 However, the discussions regarding stability of different substance classes is often qualitative. We don't have a quantitative was to compare these statements for the specific substances assessed herebroadly applicable quantitative tests are needed for stability evaluation.

In addition, encapsulation's impacts on stability need to be well established for each substance class. Encapsulation can also have impacts on the performance metrics reviewed here. The quantum yield for substances in this assessment were measured in solution, not in the powder form or embedded in plastic. This change in environment typically does not affect QDs performance, but has been known to impact perovskites. ${ }^{55}$ Perovskites suffer from quenching in powder and color segregation in mixtures (in other words, ion exchange which could muddle colors). Encapsulation also affects InP QDs. In-QDs that do 
not have excellent electron confinement (which originates from imperfect shell structure or incompatible ligands) can also have unwanted emission and loss of color purity (i.e. FWHM suffers). ${ }^{63}$ InP QDs mechanisms of degradation are not as studied in the literature as those affecting Cd-QDs. Their structural similarities between In-QDs and Cd-QDs have meant that often Cd-relevant designs for QLEDs are used. A deeper understanding of InP-specific instability can help overcome this barrier. ${ }^{63}$

In summary, these performance metrics are not perfect, but they do highlight certain essential properties of successful emissive materials. No one substance class had overall better performance than the rest, indicating that an individual synthesis can be the key to high scores.

\section{Hazard Assessment}

The hazard scores for each package were averaged (Fig. 3A) which broadly demonstrates that there is no fully innocuous package. In addition, no package stands out as less hazardous than the others. To investigate differences that could be averaged out, we chose to expand the data into a heat map (see Table S3 for specific scores). A heat map that separates hazards by type (health, environment, and physical properties) as well as by substance (red, green, and blue) demonstrates the nuances in the data (Fig. 3B).

A

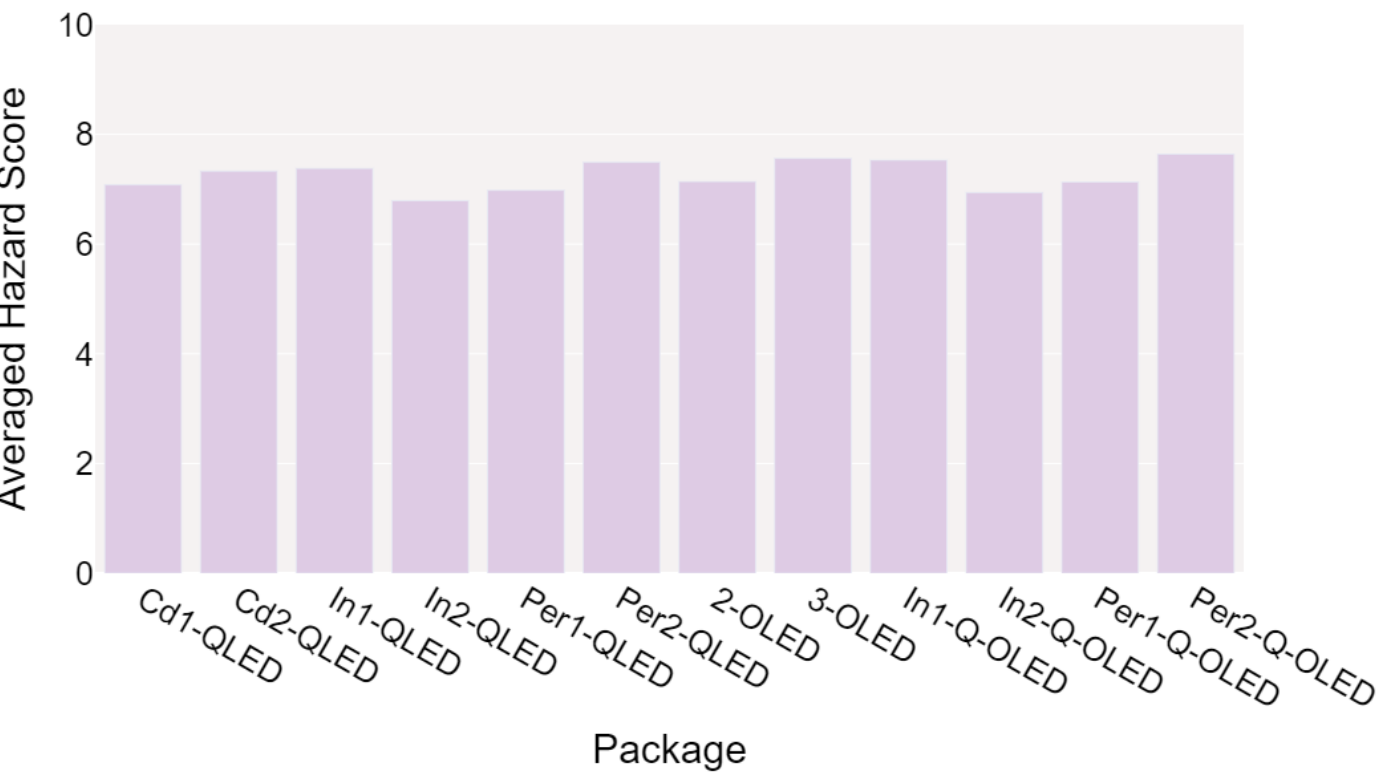




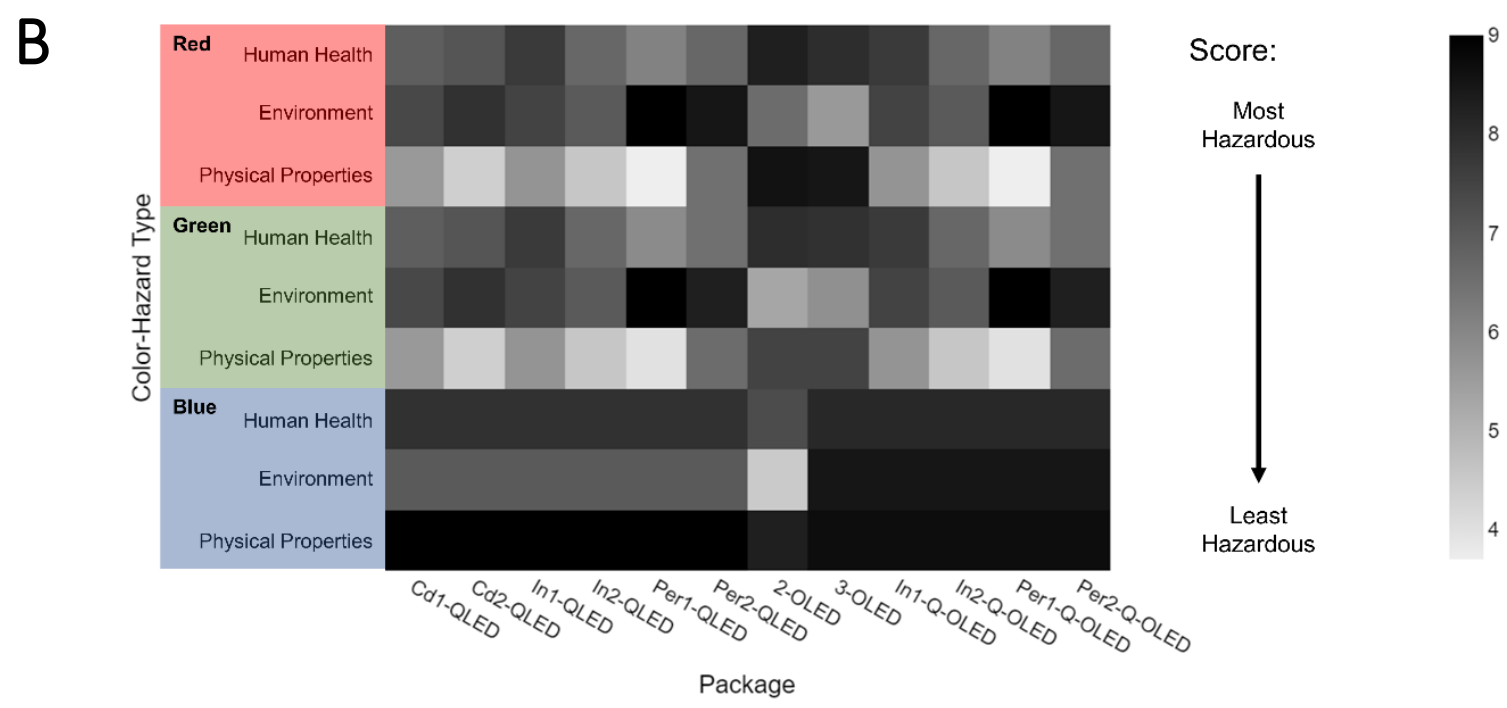

Figure 4: (A) averaged hazard assessment results and (B) hazard heat map, where dark indicates the most hazard, while light indicates the least hazard per substance (y-axis, red/green/blue) in a package (x-axis)

Touted as more sustainable than Cd-QDs in industry, ${ }^{64}$ In-QDs do not score as less hazardous in this assessment. Although In-QDs do score better than Cd-QDs in their environmental safety by 1 point, In-QDs score an average of 0.3 and 0.1 worse in health and physical properties, respectively, than CdQDs. These results contradict the Cd-QDs and In-QDs toxicity to human cells, which has been compared in a side-by-side manner by Pompa et al. ${ }^{65}$ These QDs demonstrated similar instability and ion release. ${ }^{65}$ However, because In ions were less toxic, so too were the In-QDs compared to Cd-QDs. ${ }^{65}$ These side-byside toxicity assessments are crucial, but the chemicals involved in the synthesis of QDs must also be less harmful, which is not the case.

Lead-based perovskites (Per1 packages) have low relatively health and physical properties hazards (average is 4.9 compared to the average of other packages which is 7.0). In contrast, they have a higher environmental hazard (average is 9 compared to the average of other packages which is 7.5), which roughly translates to $\mathrm{LC}_{50}$ for a $96 \mathrm{~h}$ exposure to fish that is $\leq 1 \mathrm{mg} / \mathrm{L}$. Surprisingly, the lead-free perovskite package (Per2 packages) was assessed here to be just as hazardous to the environment as the lead-containing Per1 packages. This is interesting because the drop-in replacement for lead bromide, antimony (III) bromide, is less hazardous. Therefore, one could expect Per2 to be less hazardous. However, there is increased hazard associated with the use of cesium bromide in Per2, compared to cesium carbonate in Per1. Also, various solvents in Per2 are harmful to the environment, such as 
oleylamine and octane, similar to the solvents found in Per1. In conclusion, Per2 packages employ more hazardous solvents and other precursors, increasing its average hazard. This conclusion illustrates that "lead-free" perovskites need to be studied with as much scrutiny as lead-based materials. In addition, these hazard conclusions about Per1 and Per2 focus on the inputs of the substances, the possible end-oflife impacts are not considered.

OLEDs were assessed here to be just as hazardous as the QLED alternatives. Although the OEs are associated with organic precursors, some of their inorganic precursors were the most hazardous precursors used in their synthesis. For example, palladium acetate and copper cyanide precursors in OEs (from both packages 2-OLED and 3-OLED) scored the highest hazard possible, 10. More broadly, 53\% of the precursors for OLEDs that scored 10 (most hazardous) in at least one category had metals present, while metals only made up $30 \%$ of the precursors. The $3^{\text {rd }}$ generation OLEDs (package 3-OLED), which are free of iridium, scored better or similarly as $2^{\text {nd }}$ generation OLEDs (package 2-OLED) in health and environmental hazard, but worse (by 1.7) in terms of physical properties.

The combined hazards in Figure 4 demonstrate the hazard data that was gathered from safety data sheets (SDS) for this alternatives assessment. Missing data was not represented in Fig. 4, but a lack of data has led to regrettable substitutions in the past. Data missing from this assessment is plotted in Figure 5.

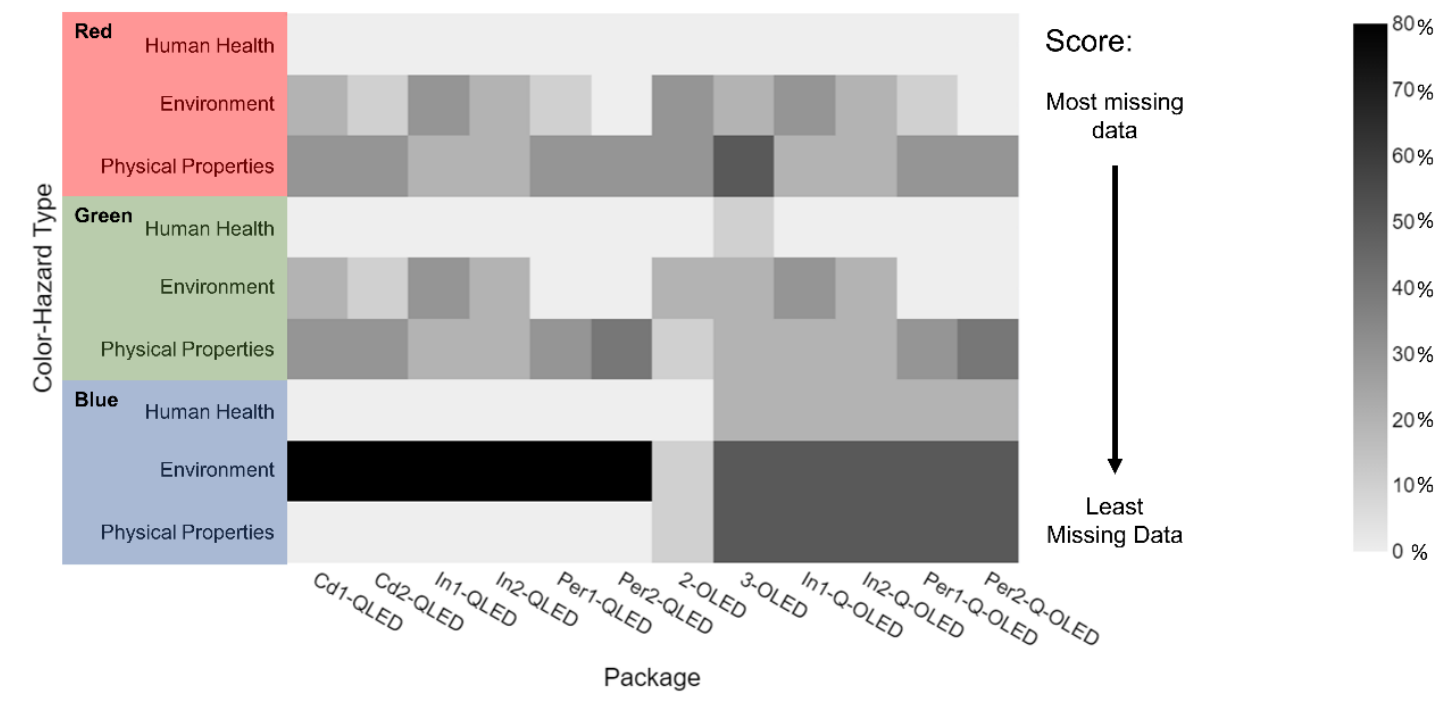

Figure 5: Initial missing hazard data results presented as a heatmap, where darker points indicate more missing data. At the maximum of the scale, $80 \%$ indicates that $80 \%$ of the precursors of a certain substance (color in the $x$ axis) in a package ( $y$-axis) had no data found in the SDS corresponding to the 
specific hazard (e.g. Environment in the $x$-axis). $0 \%$ indicates that all precursors of a substance had information regarding the specific hazard.

The least amount of missing data in Figure 5 is in the health hazard categories (see Table S4 for values). On average, only $1 \%$ of the precursors in all substances had data missing in both acute and chronic human health tests. This compares to $23-28 \%$ of the missing data for the environment and physical properties hazards. This points to the general lack of environmental hazard and stability data for many commercial chemicals. ${ }^{66}$

The largest amount of missing data is from the InGaN material, which forms the blue material of QLED displays. We hypothesize that lack of environment data is due to the high instability of the precursors (e.g. triethylgallium). These precursors decompose violently after contact with air and water and have been the cause of numerous workplace incidents. ${ }^{67,68}$ Investigation into their possible byproducts after the reaction is complete (metal oxides) also yielded missing environmental data. ${ }^{69}$ However, data gaps such as these should be addressed before choosing such an alternative over another with a more complete dataset.

Also, it is important to note the data that is missing from the health, environment, and physical properties categories. The P2OASys tool has three categories that were not integrated into Figures 4 and 5; Atmospheric Hazard, Process Factors and Life Cycle Factors. These three categories had 75-76\% missing data, which we deemed not robust enough for inclusion in the results. However, if an alternative is identified, these categories must be explored more thoroughly to avoid regrettable substitutions.

Lastly, we acknowledge a lack of missing data about the end of life of these substances. Nanomaterials (e.g. Cd-QDs, In-QDs, perovskites) are known to undergo transformations which could impact their toxicity to humans, animals, or plants. ${ }^{70}$ In addition, the encapsulation of these emissive substances for use in displays could have large impacts on their eventual behavior in the environment, and pose occupational risks to workers who tend to dismantle electronic items. ${ }^{71}$ For ease of use of this assessment by other scientists, we decided to omit these end-of-life considerations. We hypothesize that decreasing the inherent hazard of the precursors of emissive substances could translate into less concern at their end of life.

\section{Aggregation of Evaluations}

With the three separate evaluations complete, the results were combined to determine if one alternative presented an optimal combination of high performance, competitive cost, and low hazard 
(Figure 6). To do this, the packages were assigned a rank per evaluation (e.g., the best performing material received a 1, least hazardous received a 1 and the lowest cost received a 1). Then the three ranks were combined equally (Figure 6A). If a package scored a ' 3 ', this would indicate that it had the 'best'/ideal combination of performance, cost, and hazard. However, this was not the case for any package, and the best total score was 2.3 (In2-Q-OLED, Figure 6B), closely followed by 2.2 (In2-QLED) and 2.0 (2-OLED). The lowest total ranks were 1.0 (Per2-Q-OLED and Per2-QLED) and 1.1 (In1-QLED).

To demonstrate the versatility of the alternatives assessment, different weights were given to each evaluation (Figure 6C), and the scores changed (Figure 6D). With the weights being 1 for cost, 0.5 for performance, and 2 for environment, the highest possible score was 3.5. This did not change the top and bottom ranked packages mentioned above, but it did slightly increase the rank of Per1-QLED and decrease the rank of Per2-Q-OLED.

A

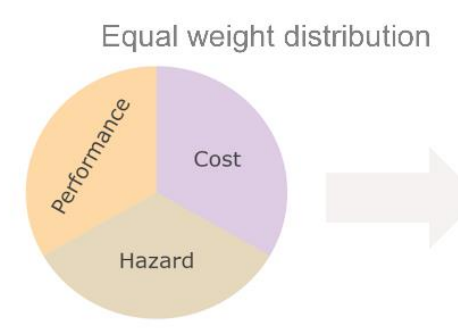

C

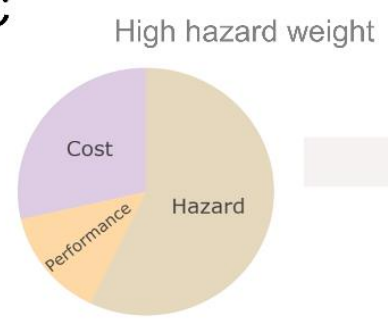

B
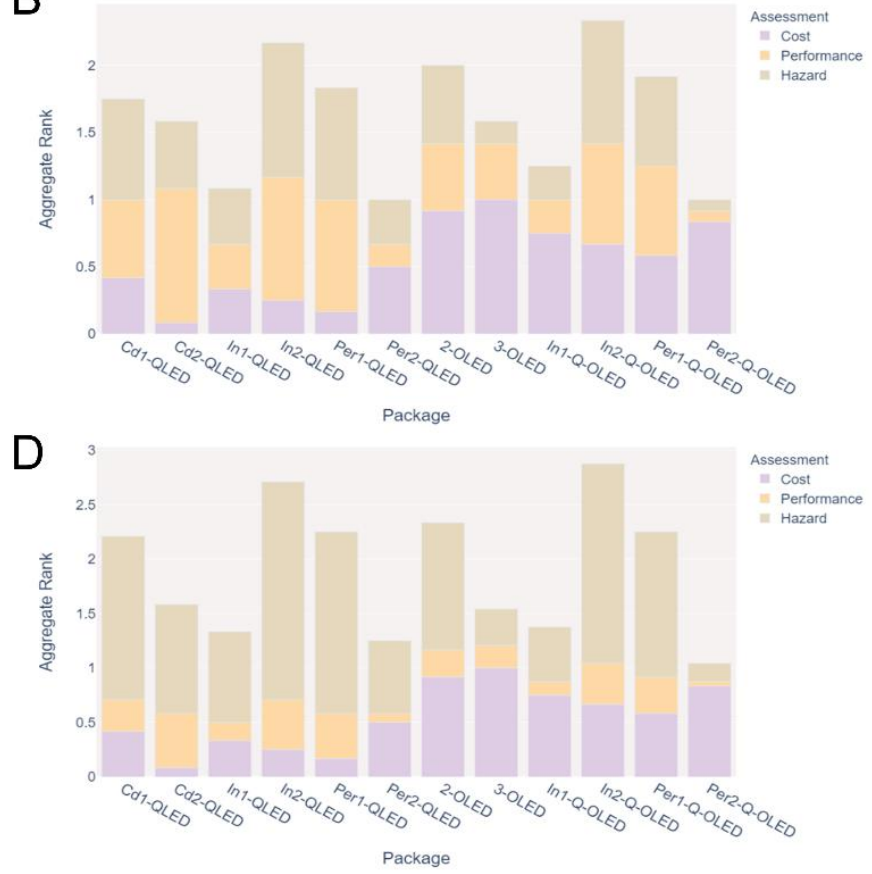

Figure 6: $(A, B)$ Combined assessments with weight of 1 for each assessment (best in a category has a score of 1$)(C, D)$ Combined assessments with (starting from the bottom) a weight of 1 for cost, 0.5 for performance, and 2 for hazard (best in the category has a score of $1,0.5$ and 2 respectively). 
These aggregate rankings both smooth out large differences in the packages (e.g. cost where variation is 5000 fold, Figure 2) while increasing the differences between packages (e.g., hazard, where all average scores are within $6 \%$ of the average). However, keeping these factors in mind, it is still possible to draw a couple of conclusions.

First, there is no substance class that is better than the others based on the findings of our assessment. The average score of all packages was 1.63, and each substance class had one package that scored above this, and another that scored below. In other words, there is no substance class with both packages having a better aggregate score than the other substance classes. This, however, can be seen as an opportunity to develop safer, cheaper, higher-performing synthesis in any substance class. The more mature substance class, Cd-QDs in QLEDs, gave very similar aggregate scores, while newer substance classes like perovskites in QLEDs gave different scores.

Second, there is variation within substance classes, except for Cd-QDs. Cd-QDs containing packages scored within 0.5 of each other. The packages representing other substance classes (In-QDs, perovskites, and OEs) scored father apart from each other. This indicates the importance of individual syntheses. Since only 12 packages were chosen for analysis, there is the possibility that a superior synthesis was overlooked. For this reason, the assessment is structured such that it can be reproduced and updated with the latest innovation in these varied substance classes.

The example given in Figure 6C,D illustrates how changing priorities can change the overall score of different packages. This set of weights could be the priorities of a company that wants to make a cheap, safe display that does not compete at the top of the line in terms of performance. This set of priorities would probably lead to more development of In1-QDs, which scored relatively well in both QLED and Q-OLEDs.

\section{Conclusions}

This alternatives assessment introduced different metrics for the emissive substance scientist to consider when developing their materials. Along with performance, cost and hazard are important considerations for the eventual manufacturers and end-users of these materials. This study highlighted the cost, performance, and hazard of emerging emissive substance classes by combining substances into packages. We found that the synthesis of the substance has a large impact on its performance and cost, while hazards (when averaged) were largely the same. Combining these results highlighted that no one 
substance class is better than the others. This was surprising to us given the excitement in the literature regarding the sustainability of perovskites ${ }^{55}$ and In-QDs. ${ }^{64}$

However, this alternatives assessment was not meant to conclusively rank all different substance classes or substances in this field, but rather to illustrate a method that could be used to assess new materials. This method has gaps in each assessment that can be filled by other researchers. In the cost assessment, we do not consider the possible impacts of encapsulation of each material, or the amount that could be wasted during manufacturing. In the performance assessment, there is no consideration of a materials stability because of a lack of standardized reporting by the primary sources. In the hazard assessment, we acknowledge that the hazard of a material is not simply the sum of its precursors and solvents. Work correlating the hazard of the precursors of a substance to the actual hazard of that substance at its end of life is necessary. Despite these gaps, this alternatives assessment did serve its original purpose of identifying possible issues with alternatives for Cd-QDs.

If this alternatives assessment had identified a preferable alternative, more research and different sustainability assessments would be needed. We designate stability metrics as well as life cycle and expanded hazard metrics, as keys to the next steps to determine the suitability for use in displays. The limitations of this study are opportunities for further study by researchers developing emissive substances as well as toxicologists and environmental scientists. 


\section{References:}

(1) Forti, V.; Balde, C. P.; Kuehr, R.; Bel, G. The Global E-Waste Monitor 2020: Quantities, Flows and the Circular Economy Potential.; United Nations University (UNU)/United Nations Institute for Training and Research (UNITAR) - co-hosted SCYCLE Programme, International Telecommunication Union (ITU) \& International Solid Waste Association (ISWA),: Bonn/Geneva/Rotterdam, 2020; pp 1-15.

(2) Lim, S.-R.; Schoenung, J. M. Human Health and Ecological Toxicity Potentials Due to Heavy Metal Content in Waste Electronic Devices with Flat Panel Displays. Journal of Hazardous Materials 2010, 177 (1), 251-259. https://doi.org/10.1016/j.jhazmat.2009.12.025.

(3) Zhao, X.; Ng, J. D. A.; Friend, R. H.; Tan, Z.-K. Opportunities and Challenges in Perovskite LightEmitting Devices. ACS Photonics 2018, 5 (10), 3866-3875. https://doi.org/10.1021/acsphotonics.8b00745.

(4) Fawell, J. Cadmium in Drinking-Water; Background document for development of WHO Guidelines for Drinking-water Quality; WHO/SDE/WSH/03.04/80/Rev/1; World Health Organization: Geneva, 2011; pp 1-16.

(5) Chen, H.-W.; Lee, J.-H.; Lin, B.-Y.; Chen, S.; Wu, S.-T. Liquid Crystal Display and Organic LightEmitting Diode Display: Present Status and Future Perspectives. Light Sci App/ 2018, 7 (3), 1716817168. https://doi.org/10.1038/Isa.2017.168.

(6) Chopra, S. S.; Theis, T. L. Comparative Cradle-to-Gate Energy Assessment of Indium Phosphide and Cadmium Selenide Quantum Dot Displays. Environ. Sci.: Nano 2017, 4 (1), 244-254. https://doi.org/10.1039/C6EN00326E.

(7) S. Chopra, S.; Bi, Y.; C. Brown, F.; L. Theis, T.; D. Hristovski, K.; Westerhoff, P. Interdisciplinary Collaborations to Address the Uncertainty Problem in Life Cycle Assessment of Nano-Enabled Products: Case of the Quantum Dot-Enabled Display. Environmental Science: Nano 2019, 6 (11), 3256-3267. https://doi.org/10.1039/C9EN00603F.

(8) Scalbi, S.; Fantin, V.; Antolini, F. Environmental Assessment of New Technologies: Production of a Quantum Dots-Light Emitting Diode. Journal of Cleaner Production 2017, 142, 3702-3718. https://doi.org/10.1016/j.jclepro.2016.10.098.

(9) Schileo, G.; Grancini, G. Lead or No Lead? Availability, Toxicity, Sustainability and Environmental Impact of Lead-Free Perovskite Solar Cells. Journal of Materials Chemistry C 2021, 9 (1), 67-76. https://doi.org/10.1039/D0TC04552G.

(10) Ke, W.; Kanatzidis, M. G. Prospects for Low-Toxicity Lead-Free Perovskite Solar Cells. Nature Communications 2019, 10 (1), 965. https://doi.org/10.1038/s41467-019-08918-3.

(11) Amasawa, E.; Ihara, T.; Ohta, T.; Hanaki, K. Life Cycle Assessment of Organic Light Emitting Diode Display as Emerging Materials and Technology. Journal of Cleaner Production 2016, 135, 13401350. https://doi.org/10.1016/j.jclepro.2016.07.025.

(12) Yeom, J.-M.; Jung, H.-J.; Choi, S.-Y.; Lee, D. S.; Lim, S.-R. Environmental Effects of the Technology Transition from Liquid-Crystal Display (LCD) to Organic Light-Emitting Diode (OLED) Display from an E-Waste Management Perspective. Int J Environ Res 2018, 12 (4), 479-488. https://doi.org/10.1007/s41742-018-0106-y.

(13) Curran, M. A. Strengths and Limitations of Life Cycle Assessment. In Background and Future Prospects in Life Cycle Assessment; Klöpffer, W., Ed.; Springer Netherlands: Dordrecht, 2014; pp 189-206. https://doi.org/10.1007/978-94-017-8697-3_6.

(14) Gallagher, M. J.; Allen, C.; Buchman, J. T.; Qiu, T. A.; Clement, P. L.; Krause, M. O. P.; Gilbertson, L. M. Research Highlights: Applications of Life-Cycle Assessment as a Tool for Characterizing Environmental Impacts of Engineered Nanomaterials. Environ. Sci.: Nano 2017, 4 (2), 276-281. https://doi.org/10.1039/C7EN90005H. 
(15) Gilbertson, L. M.; Wender, B. A.; Zimmerman, J. B.; Eckelman, M. J. Coordinating Modeling and Experimental Research of Engineered Nanomaterials to Improve Life Cycle Assessment Studies. Environ. Sci.: Nano 2015, 2 (6), 669-682. https://doi.org/10.1039/C5EN00097A.

(16) Jacobs, M. M.; Malloy, T. F.; Tickner, J. A.; Edwards, S. Alternatives Assessment Frameworks: Research Needs for the Informed Substitution of Hazardous Chemicals. Environ Health Perspect 2016, 124 (3), 265-280. https://doi.org/10.1289/ehp.1409581.

(17) Council, N. R.; Studies, D. on E. and L.; Toxicology, B. on E. S. and; Technology, B. on C. S. and; Decisions, C. on the D. and E. of S. C. S. A. F. to I. G. and I. A Framework to Guide Selection of Chemical Alternatives; National Academies Press, 2014.

(18) Gilbertson, L. M.; Ng, C. A. Evaluating the Use of Alternatives Assessment To Compare Bulk Organic Chemical and Nanomaterial Alternatives to Brominated Flame Retardants. ACS Sustainable Chem. Eng. 2016, 4 (11), 6019-6030. https://doi.org/10.1021/acssuschemeng.6b01318.

(19) Llanos, M.; Yekani, R.; Demopoulos, G. P.; Basu, N. Alternatives Assessment of Perovskite Solar Cell Materials and Their Methods of Fabrication. Renewable and Sustainable Energy Reviews 2020, 133, 110207. https://doi.org/10.1016/j.rser.2020.110207.

(20) Chen, J.; Zhang, S.; Allen, D. T.; Subramaniam, B.; Licence, P. Expectations for Manuscripts Contributing to the Field on Management of Synthetic Chemicals in ACS Sustainable Chemistry \& Engineering. ACS Sustainable Chem. Eng. 2021, 9 (9), 3376-3378. https://doi.org/10.1021/acssuschemeng.1c01134.

(21) Zhang, H.; Sui, N.; Chi, X.; Wang, Y.; Liu, Q.; Zhang, H.; Ji, W. Ultrastable Quantum-Dot LightEmitting Diodes by Suppression of Leakage Current and Exciton Quenching Processes. ACS Appl. Mater. Interfaces 2016, 8 (45), 31385-31391. https://doi.org/10.1021/acsami.6b09246.

(22) Zhu, R.; Luo, Z.; Chen, H.; Dong, Y.; Wu, S.-T. Realizing Rec. 2020 Color Gamut with Quantum Dot Displays. Opt. Express, OE 2015, 23 (18), 23680-23693. https://doi.org/10.1364/OE.23.023680.

(23) Theis, D. Display Technology. In Ullmann's Encyclopedia of Industrial Chemistry; Wiley-VCH Verlag GmbH \& Co. KGaA, Ed.; Wiley-VCH Verlag GmbH \& Co. KGaA: Weinheim, Germany, 2008; $p$ a08_603.pub2. https://doi.org/10.1002/14356007.a08_603.pub2.

(24) Carter, C. M.; Cho, J.; Glanzer, A.; Kamcev, N.; O'Carroll, D. M. Cost, Energy and Emissions Assessment of Organic Polymer Light-Emitting Device Architectures. Journal of Cleaner Production 2016, 137, 1418-1431. https://doi.org/10.1016/j.jclepro.2016.07.186.

(25) Luo, Z.; Xu, D.; Wu, S. Emerging Quantum-Dots-Enhanced LCDs. Journal of Display Technology 2014, 10 (7), 526-539. https://doi.org/10.1109/JDT.2014.2325218.

(26) Brown, R. P.; Gallagher, M. J.; Fairbrother, D. H.; Rosenzweig, Z. Synthesis and Degradation of Cadmium-Free InP and InPZn/ZnS Quantum Dots in Solution. Langmuir 2018, 34 (46), 1392413934. https://doi.org/10.1021/acs.langmuir.8b02402.

(27) Chen, Y.; Li, S.; Huang, L.; Pan, D. Low-Cost and Gram-Scale Synthesis of Water-Soluble Cu-InS/ZnS Core/Shell Quantum Dots in an Electric Pressure Cooker. Nanoscale 2014, 6 (3), 12951298. https://doi.org/10.1039/C3NR05014A.

(28) Li, C.-H. A.; Zhou, Z.; Vashishtha, P.; Halpert, J. E. The Future Is Blue (LEDs): Why Chemistry Is the Key to Perovskite Displays. Chem. Mater. 2019, 31 (16), 6003-6032. https://doi.org/10.1021/acs.chemmater.9b01650.

(29) Protesescu, L.; Yakunin, S.; Bodnarchuk, M. I.; Krieg, F.; Caputo, R.; Hendon, C. H.; Yang, R. X.; Walsh, A.; Kovalenko, M. V. Nanocrystals of Cesium Lead Halide Perovskites (CsPbX3, X $=\mathrm{Cl}, \mathrm{Br}$, and I): Novel Optoelectronic Materials Showing Bright Emission with Wide Color Gamut. Nano Lett. 2015, 15 (6), 3692-3696. https://doi.org/10.1021/nl5048779. 
(30) Zhang, J.; Yang, Y.; Deng, H.; Farooq, U.; Yang, X.; Khan, J.; Tang, J.; Song, H. High Quantum Yield Blue Emission from Lead-Free Inorganic Antimony Halide Perovskite Colloidal Quantum Dots. ACS Nano 2017, 11 (9), 9294-9302. https://doi.org/10.1021/acsnano.7b04683.

(31) Commission Delegated Directive (EU) 2015/863 of 31 March 2015 Amending Annex II to Directive 2011/65/EU of the European Parliament and of the Council as Regards the List of Restricted Substances (Text with EEA Relevance); 2015; Vol. OJ L.

(32) Hong, G.; Gan, X.; Leonhardt, C.; Zhang, Z.; Seibert, J.; Busch, J. M.; Bräse, S. A Brief History of OLEDs-Emitter Development and Industry Milestones. Adv. Mater. 2021, 33 (9), 2005630. https://doi.org/10.1002/adma.202005630.

(33) Li, T.-Y.; Wu, J.; Wu, Z.-G.; Zheng, Y.-X.; Zuo, J.-L.; Pan, Y. Rational Design of Phosphorescent Iridium(III) Complexes for Emission Color Tunability and Their Applications in OLEDs. Coordination Chemistry Reviews 2018, 374, 55-92. https://doi.org/10.1016/j.ccr.2018.06.014.

(34) Teng, J.-M.; Wang, Y.-F.; Chen, C.-F. Recent Progress of Narrowband TADF Emitters and Their Applications in OLEDs. Journal of Materials Chemistry C 2020, 8 (33), 11340-11353. https://doi.org/10.1039/D0TC02682D.

(35) Kim, K.-H.; Kim, J.-J. Origin and Control of Orientation of Phosphorescent and TADF Dyes for HighEfficiency OLEDs. Advanced Materials 2018, 30 (42), 1705600. https://doi.org/10.1002/adma.201705600.

(36) Liao, J.-L.; Rajakannu, P.; Gnanasekaran, P.; Tsai, S.-R.; Lin, C.-H.; Liu, S.-H.; Chang, C.-H.; Lee, G.H.; Chou, P.-T.; Chen, Z.-N.; Chi, Y. Luminescent Diiridium Complexes with Bridging Pyrazolates: Characterization and Fabrication of OLEDs Using Vacuum Thermal Deposition. Advanced Optical Materials 2018, 6 (11), 1800083. https://doi.org/10.1002/adom.201800083.

(37) Yang, X.; Guo, H.; Liu, B.; Zhao, J.; Zhou, G.; Wu, Z.; Wong, W.-Y. Diarylboron-Based Asymmetric Red-Emitting $\operatorname{Ir}(\mathrm{III})$ Complex for Solution-Processed Phosphorescent Organic Light-Emitting Diode with External Quantum Efficiency above 28\%. Adv. Sci. 2018, 5 (5), 1701067. https://doi.org/10.1002/advs.201701067.

(38) Shin, H.; Ha, Y. H.; Kim, H.; Kim, R.; Kwon, S.; Kim, Y.; Kim, J. Controlling Horizontal Dipole Orientation and Emission Spectrum of Ir Complexes by Chemical Design of Ancillary Ligands for Efficient Deep-Blue Organic Light-Emitting Diodes. Adv. Mater. 2019, 31 (21), 1808102. https://doi.org/10.1002/adma.201808102.

(39) Wu, T.-L.; Huang, M.-J.; Lin, C.-C.; Huang, P.-Y.; Chou, T.-Y.; Chen-Cheng, R.-W.; Lin, H.-W.; Liu, R.S.; Cheng, C.-H. Diboron Compound-Based Organic Light-Emitting Diodes with High Efficiency and Reduced Efficiency Roll-Off. Nature Photon 2018, 12 (4), 235-240. https://doi.org/10.1038/s41566-018-0112-9.

(40) Zhang, Y.; Ran, Q.; Wang, Q.; Liu, Y.; Hänisch, C.; Reineke, S.; Fan, J.; Liao, L. High-Efficiency Red Organic Light-Emitting Diodes with External Quantum Efficiency Close to 30\% Based on a Novel Thermally Activated Delayed Fluorescence Emitter. Adv. Mater. 2019, 31 (42), 1902368. https://doi.org/10.1002/adma.201902368.

(41) Lin, T.-A.; Chatterjee, T.; Tsai, W.-L.; Lee, W.-K.; Wu, M.-J.; Jiao, M.; Pan, K.-C.; Yi, C.-L.; Chung, C.L.; Wong, K.-T.; Wu, C.-C. Sky-Blue Organic Light Emitting Diode with 37\% External Quantum Efficiency Using Thermally Activated Delayed Fluorescence from Spiroacridine-Triazine Hybrid. Adv. Mater. 2016, 28 (32), 6976-6983. https://doi.org/10.1002/adma.201601675.

(42) Nanosys. Review of Impact of Potential New RoHS Substance Restrictions for Indium-Phosphide. Nanosys November 2019.

(43) Li, Y.; Hou, X.; Dai, X.; Yao, Z.; Lv, L.; Jin, Y.; Peng, X. Stoichiometry-Controlled InP-Based Quantum Dots: Synthesis, Photoluminescence, and Electroluminescence. J. Am. Chem. Soc. 2019, 141 (16), 6448-6452. https://doi.org/10.1021/jacs.8b12908. 
(44) Jang, E.; Jun, S.; Jang, H.; Lim, J.; Kim, B.; Kim, Y. White-Light-Emitting Diodes with Quantum Dot Color Converters for Display Backlights. Advanced Materials 2010, 22 (28), 3076-3080. https://doi.org/10.1002/adma.201000525.

(45) Shenai-Khatkhate, D. V.; Goyette, R. J.; DiCarlo Jr., R. L.; Dripps, G. Environment, Health and Safety Issues for Sources Used in MOVPE Growth of Compound Semiconductors. Journal of Crystal Growth 2004, 272 (1), 816-821. https://doi.org/10.1016/j.jcrysgro.2004.09.007.

(46) Volz, D.; Wallesch, M.; Fléchon, C.; Danz, M.; Verma, A.; M. Navarro, J.; M. Zink, D.; Bräse, S.; Baumann, T. From Iridium and Platinum to Copper and Carbon: New Avenues for More Sustainability in Organic Light-Emitting Diodes. Green Chemistry 2015, 17 (4), 1988-2011. https://doi.org/10.1039/C4GC02195A.

(47) Sun, Y.; Jiang, Y.; Sun, X. W.; Zhang, S.; Chen, S. Beyond OLED: Efficient Quantum Dot LightEmitting Diodes for Display and Lighting Application. The Chemical Record 2019, 19 (8), 17291752. https://doi.org/10.1002/tcr.201800191.

(48) Yang, C.; Zhuang, B.; Lin, J.; Wang, S.; Liu, M.; Jiang, N.; Chen, D. Ultrastable Glass-Protected AllInorganic Perovskite Quantum Dots with Finely Tunable Green Emissions for Approaching Rec. 2020 Backlit Display. Chemical Engineering Journal 2020, 398, 125616.

https://doi.org/10.1016/j.cej.2020.125616.

(49) Bechu, A.; Griffin, H. Emissive Substance Spectral Overlap with Rec. 2020, 2021. https://www.mooresresearch.org/alternativeassessmentqdot

(50) Chen, H.-W.; Zhu, R.-D.; He, J.; Duan, W.; Hu, W.; Lu, Y.-Q.; Li, M.-C.; Lee, S.-L.; Dong, Y.-J.; Wu, S.T. Going beyond the Limit of an LCD's Color Gamut. Light: Science \& Applications 2017, 6 (9), e17043-e17043. https://doi.org/10.1038/lsa.2017.43.

(51) Wang, T. Topical Review: Development of Overgrown Semi-Polar GaN for High Efficiency Green/Yellow Emission. Semicond. Sci. Technol. 2016, 31, 093003. https://doi.org/10.1088/02681242/31/9/093003.

(52) Regulation (EC) No 1907/2006 of the European Parliament and of the Council of 18 December 2006 Concerning the Registration, Evaluation, Authorisation and Restriction of Chemicals (REACH), Establishing a European Chemicals Agency, Amending Directive 1999/45/EC and Repealing Council Regulation (EEC) No 793/93 and Commission Regulation (EC) No 1488/94 as Well as Council Directive 76/769/EEC and Commission Directives 91/155/EEC, 93/67/EEC, 93/105/EC and 2000/21/EC; 2006; Vol. 396.

(53) P2OASys - Tool - About https://p2oasys.turi.org/GetStarted/About/about.html (accessed 2021 $01-11)$.

(54) Chen, Z.; Yan, S.; Danesh, C. MicroLED Technologies and Applications: Characteristics, Fabrication, Progress, and Challenges. J. Phys. D: Appl. Phys. 2021, 54 (12), 123001. https://doi.org/10.1088/1361-6463/abcfe4.

(55) Wang, X.; Bao, Z.; Chang, Y.-C.; Liu, R.-S. Perovskite Quantum Dots for Application in High Color Gamut Backlighting Display of Light-Emitting Diodes. ACS Energy Lett. 2020, 5 (11), 3374-3396. https://doi.org/10.1021/acsenergylett.0c01860.

(56) Critical Raw Materials Resilience: Charting a Path towards Greater Security and Sustainability-; Communication COM(2020) 474 final; European Commission: Brussels, 2020.

(57) Recommendation ITU-R BT.2020-2: Parameter Values for Ultra-High Definition Television Systems for Production and International Programme Exchange. International Telecommunication Union 2015.

(58) Yu, W. W.; Qu, L.; Guo, W.; Peng, X. Experimental Determination of the Extinction Coefficient of CdTe, CdSe, and CdS Nanocrystals. Chemistry of Materials 2003, 15 (14), 2854-2860.

(59) Chen, L.; You, H.; Yang, C.; Zhang, X.; Qin, J.; Ma, D. Tuning the Saturated Red Emission: Synthesis, Electrochemistry and Photophysics of 2-Arylquinoline Based Iridium(III) Complexes and Their 
Application in OLEDs. J. Mater. Chem. 2006, 16 (32), 3332-3339.

https://doi.org/10.1039/B605783G.

(60) Lee, J.-H.; Chen, C.-H.; Lee, P.-H.; Lin, H.-Y.; Leung, M.; Chiu, T.-L.; Lin, C.-F. Blue Organic LightEmitting Diodes: Current Status, Challenges, and Future Outlook. J. Mater. Chem. C 2019, 7 (20), 5874-5888. https://doi.org/10.1039/C9TC00204A.

(61) Liu, Z.; Lin, C.-H.; Hyun, B.-R.; Sher, C.-W.; Lv, Z.; Luo, B.; Jiang, F.; Wu, T.; Ho, C.-H.; Kuo, H.-C.; He, J.-H. Micro-Light-Emitting Diodes with Quantum Dots in Display Technology. Light Sci App/ 2020, 9 (1), 83. https://doi.org/10.1038/s41377-020-0268-1.

(62) Moon, H.; Lee, C.; Lee, W.; Kim, J.; Chae, H. Stability of Quantum Dots, Quantum Dot Films, and Quantum Dot Light-Emitting Diodes for Display Applications. Adv. Mater. 2019, 31 (34), 1804294. https://doi.org/10.1002/adma.201804294.

(63) Wu, Z.; Liu, P.; Zhang, W.; Wang, K.; Sun, X. W. Development of InP Quantum Dot-Based LightEmitting Diodes. ACS Energy Lett. 2020, 5 (4), 1095-1106. https://doi.org/10.1021/acsenergylett.9b02824.

(64) Ko, Y.-H.; Prabhakaran, P.; Choi, S.; Kim, G.-J.; Lee, C.; Lee, K.-S. Environmentally Friendly Quantum-Dot Color Filters for Ultra-High-Definition Liquid Crystal Displays. Sci Rep 2020, 10 (1), 15817. https://doi.org/10.1038/s41598-020-72468-8.

(65) Brunetti, V.; Chibli, H.; Fiammengo, R.; Galeone, A.; Ada Malvindi, M.; Vecchio, G.; Cingolani, R.; L. Nadeau, J.; Paolo Pompa, P. InP/ZnS as a Safer Alternative to CdSe/ZnS Core/Shell Quantum Dots : In Vitro and in Vivo Toxicity Assessment. Nanoscale 2013, 5 (1), 307-317. https://doi.org/10.1039/C2NR33024E.

(66) Ingre-Khans, E.; Ågerstrand, M.; Beronius, A.; Rudén, C. Transparency of Chemical Risk Assessment Data under REACH. Environ. Sci.: Processes Impacts 2016, 18 (12), 1508-1518. https://doi.org/10.1039/C6EM00389C.

(67) Wojcik, S. M. OSHA issues $\$ 28,000$ in fines for Upper Macungie lab explosion https://www.mcall.com/news/local/parkland/mc-upper-macungie-cyoptics-osha-report20170725-story.html (accessed 2021 -06 -10).

(68) Dow Chemical Worker Dies After Plant Fire https://cen.acs.org/articles/91/i42/Dow-ChemicalWorker-Dies-Plant.html (accessed 2021 -06 -10).

(69) PubChem. Indium oxide https://pubchem.ncbi.nlm.nih.gov/compound/150905 (accessed 2021 $06-10)$.

(70) Zhang, J.; Guo, W.; Li, Q.; Wang, Z.; Liu, S. The Effects and the Potential Mechanism of Environmental Transformation of Metal Nanoparticles on Their Toxicity in Organisms. Environmental Science: Nano 2018, 5 (11), 2482-2499. https://doi.org/10.1039/C8EN00688A.

(71) J. Gallagher, M.; T. Buchman, J.; A. Qiu, T.; Zhi, B.; Y. Lyons, T.; M. Landy, K.; Rosenzweig, Z.; L. Haynes, C.; Howard Fairbrother, D. Release, Detection and Toxicity of Fragments Generated during Artificial Accelerated Weathering of CdSe/ZnS and CdSe Quantum Dot Polymer Composites. Environmental Science: Nano 2018, 5 (7), 1694-1710. https://doi.org/10.1039/C8EN00249E.

Funding:

We thank the Natural Science and Engineering Research Council of Canada (NSERC) Discovery Grant and Discovery Accelerator Supplement, the McGill Sustainability Systems Initiative (MSSI), the Fonds de Recherche du Québec - Nature et Technologies (FRQNT) Graduate Scholarship program (AB), the Canada Research Chair Program, the Dr. Lawrence Light Graduate Fellowships in Sustainability and McGill University for their financial support. 


\section{Acknowledgements:}

We would like to thank Harrisonn Griffin for his idea of creating a web-based tool for the Rec.2020 Overlap. His technical expertise was key to the realization of the tool. We would also like to thank Mariana Llanos for her work with the initial database. 